\title{
Positive emotion regulation strategies as mediators in depression and generalized anxiety disorder symptoms: A Transdiagnostic framework investigation
}

\author{
Imaneh Abasi $^{1} \cdot$ Giti Shams ${ }^{2}$ - Belén Pascual-Vera3 ${ }^{3}$ - Irena Milosevic ${ }^{4}$ Maryam Bitarafan ${ }^{1}$. \\ Sepideh Ghanadanzadeh ${ }^{5} \cdot$ Maryam Talebi Moghaddam $^{6}$
}

Accepted: 14 January 2021

(C) The Author(s), under exclusive licence to Springer Science+Business Media, LLC part of Springer Nature 2021

\begin{abstract}
Positive emotion regulation (ER) strategies may contribute to the development and maintenance of generalized anxiety disorder (GAD) and depression; nonetheless, the underlying and transdiagnostic mechanisms are still unknown. To examine: 1) the mediating role of positive ER strategies in the relationship between ER deficits and experiential avoidance in GAD and depression symptoms; 2) differences in positive ER strategies among participants with elevated symptoms of GAD, depression, depression comorbid with GAD, and a nonclinical sample. 532 Tehran University students completed questionnaires assessing experiential avoidance, deficits in ER, positive ER strategies (dampening, positive rumination), and GAD and depression symptoms. Differences were assessed in four groups: GAD, depression, depression comorbid with GAD, and nonclinical. Data were analysed with path analysis and MANCOVA. Dampening and positive rumination mediated the relationship between deficits in ER and experiential avoidance in participants with depression and GAD symptoms. As for the between groups comparisons, the comorbid group obtained the highest scores on dampening and the nonclinical group the lowest. By contrast, nonclinical participants had the highest scores on positive rumination, whereas the comorbid group had the lowest. Positive ER strategies might be transdiagnostic variables. Dampening was more associated with psychopathology than positive rumination, suggesting that this strategy could be more clearly considered a transdiagnostic factor. Future studies with clinical samples must support this suggestion.
\end{abstract}

Keywords Transdiagnostic $\cdot$ Dampening $\cdot$ Positive rumination $\cdot$ Experiential avoidance $\cdot$ Depression $\cdot$ Generalized anxiety disorder

Giti Shams

shamsgit@tums.ac.ir; gkshams@yahoo.com

1 Department of Clinical Psychology, School of Medicine, Shahid Beheshti University of Medical Sciences, Tehran, Iran

2 Department of Psychiatry, Tehran University of Medical Science, Roozbeh Hospital, South Kargar Avenue, Tehran 13337, Iran

3 Department of Sociology and Psychology, University of Zaragoza, Zaragoza, Spain

4 Department of Psychiatry and Behavioural Neurosciences, McMaster University, Hamilton, Ontario, Canada

5 Department of Clinical Psychology, University of Social Welfare and Rehabilitation Sciences, Tehran, Iran

6 Department of Biostatistics, University of Social Welfare and Rehabilitation Sciences, Tehran, Iran
Difficulties with emotion regulation (ER) are intertwined with a wide range of psychopathology including social anxiety disorder (Dixon et al., 2020), depression (Dryman \& Heimberg, 2018), bipolar disorder (Dodd, Lockwood, Mansell, \& Palmier-Claus, 2019), generalized anxiety disorder (O'Toole, Renna, Mennin, \& Fresco, 2019), borderline personality disorder (Chapman, 2019) and many other psychiatric disorders (Sloan et al., 2017). Previous studies have paid much attention to negative ER, whereas the role of positive ER has received little consideration. However, recently researchers are becoming more aware of the importance of positive emotion and its regulation in both healthy and clinical populations (du Pont, Welker, Gilbert, \& Gruber, 2016; Silton et al., 2020).

Positive emotion can have adaptive benefits, such as broadened attentional control (Fredrickson \& Branigan, 2005), improved physical health (Moskowitz, Epel, \& Acree, 2008), better cooperation with others (Rand, Kraft-Todd, \& Gruber, 
2015), and a buffer against psychopathology (Bonanno, PatHorenczyk, \& Noll, 2011). Importantly, there is increasing evidence that responses to positive emotion are linked to the development and maintenance of emotional disorders. Some of these strategies are used to increase positive mood and some of them are used to decrease positive mood; they are called savoring/positive rumination and dampening, respectively (Wood, Heimpel, \& Michela, 2003). Positive rumination is defined as "the tendency to respond to positive affective states with recurrent thoughts about positive self-qualities, positive affective experience, and one's favorable life circumstances," (Feldman, Joormann, \& Johnson, 2008, p. 509), and dampening is defined as the tendency to respond to positive mood states using mental strategies to reduce the intensity and duration of such states (Feldman et al., 2008). Many researchers have shown that dampening and positive rumination are associated with emotional disorders, especially depression and anxiety (Eisner, Johnson, \& Carver, 2009; Nelis, Holmes, $\&$ Raes, 2015). However, the underlying mechanisms of positive ER strategies are not well understood. Several authors argue that the similar affective disturbances in negative ER may be responsible for positive ER. For example, there is accumulating evidence that the same basic mechanism may give rise to both positive and negative emotion (Williams, Dalgleish, Karl, \& Kuyken, 2014).

Taken together, previous studies suggest examining the putative role of positive ER strategies in GAD and depression. Experiential avoidance (EA) is defined as the unwillingness to remain in contact with inconvenient private experiences (including bodily sensations, emotions, thoughts, memories, and behavioural predispositions) and the effort to avoid them (Hayes et al., 2004). EA has been hypothesized to have a crucial role in the etiology and maintenance of a range of psychological disorders, especially anxiety and depression (Chawla \& Ostafin, 2007; Hayes, 2004), and has been shown to have a predisposing and trait-like role in the prediction of anxiety disorders (Kirk, Meyer, Whisman, Deacon, \& Arch, 2019). On the other hand, deficits in emotion regulation (i.e., non-acceptance of emotional responses, such as difficulties engaging in goal directed behaviours, impulse control difficulties, and lack of emotional awareness) limit access to ER strategies, and lack of emotional clarity as an underlying construct may affect both positive and negative emotion regulation strategies. Based on this, we hypothesized that deficits in ER and experiential avoidance, which are transdiagnostic processes across a range of emotional disorders (Cludius, Mennin, \& Ehring, 2020; Spinhoven, Drost, de Rooij, van Hemert, \& Penninx, 2014), would be significantly related to different kinds of positive ER strategies (as mediators) which, in turn, would be associated with emotional disorders (depression and GAD). Among emotional disorders, depression and GAD are highly comorbid, and their comorbidity is associated with lower quality of life (Zhou et al., 2017). Furthermore,
GAD is a debilitating condition, and its treatment is less efficacious compared to other anxiety disorders (Seager, Mennin, \& Aldao, 2019). Consequently, understanding how different positive ER strategies are related to depression and GAD could provide notable and useful implications for the conceptualization and treatment of emotional disorders. In other words, if positive ER strategies are mediating the relationship between deficits in ER and experiential avoidance with depression and GAD symptoms, we could speculate that psychological treatments that target the negative emotions of individuals with emotional disorders may also be beneficial in targeting their positive emotions.

The specific objectives of the current study were twofold: first, to assess the mediating role of positive ER strategies (dampening and positive rumination) in the relationship between deficits in ER and experiential avoidance with depression and GAD symptoms, and second, to assess differences in dampening and positive rumination between individuals with depression comorbid with GAD, GAD, and depression. We hypothesized that individuals with comorbid GAD and depression experience more dampening and positive rumination than individuals with GAD only or with depression only. We expected that assessing responses to positive emotion would yield a more complete picture of these affective disorders. It may also determine which of the ER strategies is specific and which of them can be considered a transdiagnostic processes.

\section{Method}

\section{Participants}

A total sample of 535 students $(51.8 \%$ male) at Tehran University was recruited. All participants were aged between 18 and $55(M=25.32, S D=4.94)$ years. With respect to marital status, $80.2 \%$ of the participants were single and $19.8 \%$ were married. In terms of educational level, $49.2 \%, 43.9 \%$, and $6.9 \%$ of participants were Bachelor, Master's, and Ph.D. students, respectively. In order to test our second objective, four groups of participants were established: GAD group $(n=$ $28)$, depression group $(n=60)$, depression comorbid with GAD group $(n=46)$, and nonclinical group $(n=396)$. The clinical groups were established based on the cut-off scores on the BDI-II and GAD-7. Participants with GAD-7 $\geq 10$ and BDI-II $<14$ were grouped as GAD; participants with GAD-7 $<10$ and $\mathrm{BDI}-\mathrm{II} \geq 14$ were grouped as depression; and participants with GAD-7>10 and BDI-II > 29 were grouped as depression comorbid with GAD (Beck, Steer, \& Carbin, 1988; Spitzer, Kroenke, Williams, \& Löwe, 2006). There were no significant differences between groups in age, $F$ (3, $531)=1.76, p=.15$; gender, $\chi 2=6.21, p=.10$; and education, $\chi 2=9.51, p=.39$, but differences were observed in marital status $(\chi 2=57.33, p=.001)$, with more participants in the 
MDD group identifying as single $(94.2 \%)$ compared to participants in the nonclinical $(76.5 \%)$, depression comorbid with GAD $(88.1 \%)$, and GAD $(75.0 \%)$ groups.

\section{Measures}

\section{Beck Depression Inventory-II (BDI-II)}

The BDI-II is a self-report 21-item questionnaire that assesses severity of depression (Beck et al., 1988; Iranian version: Ghassemzadeh, Mojtabai, Karamghadiri, \& Ebrahimkhani, 2005). Each item is rated on a 4-point Likert-type scale ranging from 0 to 3 . The BDI-II has demonstrated internal consistency of .86 and .81 in psychiatric and non-psychiatric populations, respectively, as well as satisfactory stability and validity (Beck et al., 1988). The Persian version of the BDI-II has demonstrated good internal consistency $(\alpha=.87)$ and acceptable test-retest reliability $(r=.74)$ in Iran, as well as a strong relationship with the Automatic Thoughts Questionnaire, $r=.77$ (Ghassemzadeh et al., 2005). A cut off score of 14 has been established for diagnosis of depression (Beck et al., 1988). Cronbach's alpha of the BDI-II in the present study was .92 .

\section{Generalized Anxiety Disorder 7-Item Scale (GAD-7)}

The GAD-7 is a self-report 7-item questionnaire that assesses the severity of generalized anxiety disorder. The items are scored on a 0 (not at all) to 3 (nearly every day) Likert scale (Spitzer et al., 2006; Iranian version: Omani-Samani, Maroufizadeh, Ghaheri, \& Navid, 2018). The GAD-7 has shown excellent internal consistency $(\alpha=.92)$ and good testretest reliability $(r=.83)$, and the cut-off score of 10 has been demonstrated with optimized sensitivity $(89 \%)$ and specificity (82\%) for GAD (Spitzer et al., 2006). The GAD-7 has demonstrated satisfactory reliability $(r=.88)$ and validity in the Iranian population (Omani-Samani et al., 2018): correlation coefficients were .78 and .52 with the anxiety and depression subscales of the Hospital Anxiety and Depression Scale, respectively, and .60 with the Penn State Worry Questionnaire. Its Cronbach's alpha in the present study was .90 .

\section{Responses to Positive Affect Questionnaire (RPA)}

The RPA is a 17-item self-report questionnaire that assesses positive ER strategies (Feldman et al., 2008; Iranian version: Abasi, Feldman, Farazmand, Pourshahbaz, \& Sarichloo, 2018). It is rated on a 4-point Likert-type scale ranging from 0 (almost never) to 3 (almost always). The original form of the RPA yielded three subscales (emotion-focus, dampening, and self-focus). The internal consistency of the RPA subscales is reported to be $.76, .79$, and .73 , respectively, and the RPA has shown satisfactory construct and discriminant validity
(Feldman et al., 2008). The Iranian version of the RPA has yielded two factors: positive rumination (e.g., "Think about how you feel up for doing everything") and dampening (e.g., "Think "My streak of luck is going to end soon"). The internal consistency of these factors is reported to be .87 and .77 and test retest reliability .81 and .83 , respectively. ER strategies were significantly correlated with the Beck Depression Inventory (positive rumination: $r=.2$; dampening: $r=.39$ ) and the Social Interaction Anxiety Scale (positive rumination: $r=.15$; dampening: $r=.36$ ) (Abasi et al., 2018). Cronbach's alpha of positive rumination and savoring in the present study were .90 and .85 , respectively.

\section{Acceptance and Action Questionnaire-II (AAQ-II)}

The AAQ-II is a 10-item self-report questionnaire that assesses the tendency to evaluate unwanted thoughts and feelings negatively, not accept them, and try to alter or escape from them (Bond et al., 2011; Iranian version: Abasi, Fti, Molodi, \& Zarabi, 2013). It is scored on a Likert scale from 1 (never true) to 7 (always true). The original version of the AAQ-II showed good internal consistency (.81) and acceptable test-retest reliability (.79), respectively (Bond et al., 2011). The Iranian version of the AAQ-II demonstrated acceptable-to-good internal consistency (.76 to .82) and convergent validity with symptoms of depression and anxiety $(.44$ to .81) in nonclinical and clinical samples (Abasi et al., 2013). Cronbach's alpha of the AAQ-II in the present study was .89.

\section{Difficulties in Emotion Regulation Scale (DERS)}

The DERS is a 36 -item self-report scale that evaluates individuals' typical tendencies toward ER (Gratz \& Roemer, 2004; Iranian version: Asgari, Pasha, \& Aminiyan, 2009). It includes six subscales, including 1- Non-acceptance of emotional responses, 2- Difficulties engaging in goal directed behaviors, 3- Impulse control difficulties, 4- Lack of emotional awareness, 5- Limited access to ER strategies, and 6- Lack of emotional clarity. The DERS has demonstrated good internal consistency ( $\alpha=$ higher than .80 for each subscale) and testretest reliability, $r=.88$ (Gratz \& Roemer, 2004). The Iranian version of the DERS has demonstrated good internal consistency reliability (.86) and was shown to be significantly correlated with the Zuckerman-Kuhlman Personality Questionnaire ( $r=.26$; Asgari et al., 2009). Cronbach's alpha of the DERS in the present study was .96.

\section{Procedure}

Participants were recruited from the student population at Tehran University. The sample was selected via stratified sampling. Each college within the University was considered a stratum. Within each college, approximately $50 \%$ male and 
$50 \%$ female participants were selected randomly. All colleges of Tehran University were included in the study. Participants were informed about the study's general purpose and assessment procedure, and asked for their explicit consent to participate. Once the informed consent was signed, participants were scheduled to attend an assessment session where they were provided with a booklet containing study measures. The study received the approval of the University Ethics Committee.

\section{Data Analysis}

The analysis strategy included three steps. In the first step, we detected and deleted outliers and assessed the normality and multicollinearity of the variables. We then performed correlations between variables to ensure that the proposed model of correlations among variables is identifiable. In this stage, SPSS-23 was used for analysis (George \& Mallery, 2016). In the second step, we performed path analysis. We used the maximum likelihood estimator. Model fit was evaluated using a chi-square test and other fit indices such as the root mean square error of approximation (RMSEA), the comparative fit index (CFI), and goodness-of-fit index (GFI). These indices were considered good if they fulfilled the following criteria: RMSEA $<1$, GFI and CFI both $\geq 0.95$ (Meyers, Gamst, \& Guarino, 2016). AMOS 23.0 was used to analyze the path analysis (Arbuckle, 2014). In the third step, we performed multivariate analysis of variance (MANOVA) to evaluate the differences in positive ER strategies between the four groups (nonclinical, GAD, depression, and depression comorbid with GAD). SPSS-23 was used for this step (George \& Mallery, 2016).

\section{Results}

\section{Preliminary Data: Associations among Emotional Symptoms, Experiential Avoidance, Deficits in Emotion Regulation, and Positive Emotion Regulation Strategies}

Descriptive statistics (means and standard deviations) and correlations among variables are presented in Table 1. Correlations between depression, generalized anxiety, dampening, positive rumination, deficits in ER, and experiential avoidance were significant $(p<.01)$. Dampening correlated positively with depression, generalized anxiety, difficulties in emotion regulation, and experiential avoidance, indicating this strategy is maladaptive. Positive rumination correlated negatively with depression, generalized anxiety, difficulties in emotion regulation, and experiential avoidance, indicating this strategy may be adaptive. The strongest correlations were between both dampening and positive rumination with
Table 1 Means, Standard Deviations, and Correlations Among Study Variables

\begin{tabular}{lllllllll}
\hline & $M$ & $S D$ & 1 & 2 & 3 & 4 & 5 & 6 \\
\hline 1. DS & 12.12 & 10.26 & 1 & & & & & \\
2. GAS & 7.60 & 5.22 & $.76^{* *}$ & 1 & & & & \\
3. DAM & 11.45 & 4.25 & $.66^{* *}$ & $.57^{* *}$ & 1 & & & \\
4. PR & 27.48 & 5.84 & $-.48^{* *}$ & $-.42^{* *}$ & $-.37^{* *}$ & 1 & & \\
5. DER & 84.35 & 27.08 & $.77^{* *}$ & $.73^{* *}$ & $.68^{* *}$ & $-.46^{* *}$ & 1 & \\
6. EA & 31.40 & 11.27 & $.74^{* *}$ & $.72^{* *}$ & $.60^{* *}$ & $-.37^{* *}$ & $.78^{* *}$ & 1
\end{tabular}

Note: DS = depression symptoms; GAS = generalized anxiety symptoms; $\mathrm{DAM}=$ dampening $; \mathrm{PR}=$ positive rumination $; \mathrm{DER}=$ deficits in emotion regulation; $\mathrm{EA}=$ experiential avoidance

$* * p<.01$

depression. In order to assess multicollinearity, the variance inflation factor (VIF) and tolerance parameter were evaluated for the variables. VIF for all variables was less than 3 and tolerance was more than .1, indicating there is no multicollinearity (Meyers et al., 2016). The skewness and kurtosis of all variables were less than 1.5 , which is an acceptable range and indicates the normality of the data (Meyers et al., 2016).

\section{Mediating Role of Positive Emotion Regulation Strategies in the Relationship between Deficits in Emotion Regulation and Experiential Avoidance}

In order to assess the mediating role of dampening and positive rumination in the relationship between deficits in emotion regulation and experiential avoidance with generalized anxiety and depression symptoms, a path analysis was run. The model indices were acceptable, $\chi^{2} / \mathrm{df}=3.40$, GFI $=.99$, $\mathrm{CFI}=.99, \mathrm{RMSEA}=.06$ (Fig. 1).

\section{Direct Effects}

According to the results of the path analysis, these direct paths were significant as follows: deficits in ER had a direct impact on dampening $(\beta=.53, p<.001)$, positive rumination $(\beta=-.42, p<.001)$, depression $(\beta=.36, p<.001)$, and generalized anxiety $(\beta=.35$, $p<.001)$; experiential avoidance had a direct impact on dampening $(\beta=.19, p<.001)$, depression $(\beta=.30$, $p<.001)$, and generalized anxiety $(\beta=.36, p<.001)$; dampening had a direct impact on depression $(\beta=.19$, $p<.001)$, and generalized anxiety $(\beta=.08, p<.04)$; and positive rumination had a direct impact on depression $(\beta=-.11, p<.001)$ and generalized anxiety $(\beta=-.09$, $p<.004)$. However, the direct impact of experiential avoidance on positive rumination was not significant. 


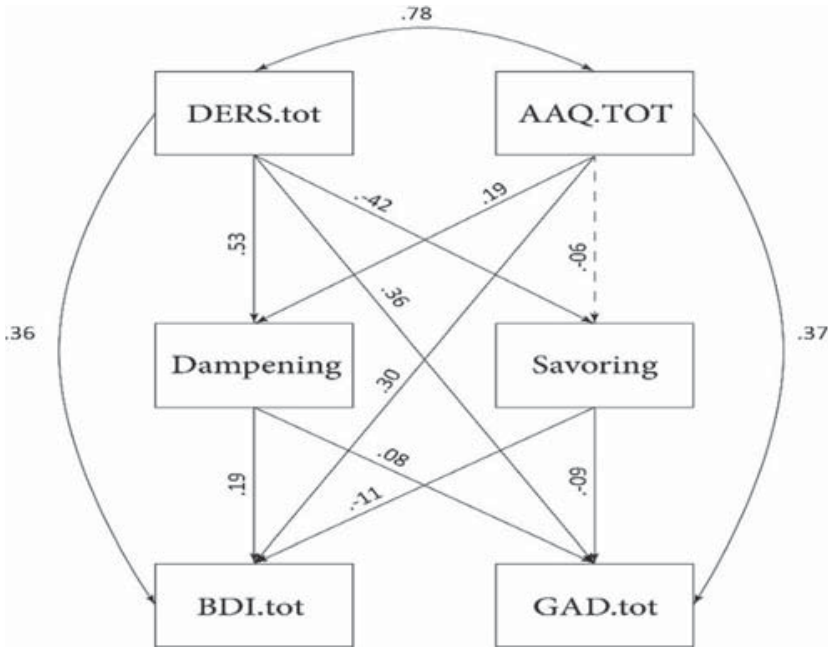

Fig. 1 Results of path analysis of direct and indirect effects of deficits in emotion regulation and experiential avoidance on depression and gad symptoms with the mediating role of dampening and positive rumination. Note. AAQ = Acceptance and Action Questionnaire-II; DERS $=$ Difficulties in Emotion Regulation Scale; BDI $=$ Beck Depression Inventory-II; GAD = Generalized Anxiety Disorder

\section{Mediation Effects}

Results indicated that deficits in ER have an indirect impact on depression $(\beta=.14)$ and generalized anxiety $(\beta=.07)$ via dampening and positive rumination, and experiential avoidance has an indirect impact on depression $(\beta=.04)$ and generalized anxiety $(\beta=.02)$ via dampening and positive rumination. These results indicate that ER strategies (dampening and positive rumination) mediate the relationship between deficits in ER and experiential avoidance with depression and generalized anxiety.

\section{Differences in Positive Emotion Regulation Strategies across GAD, Depression, Depression Comorbid with GAD, and Nonclinical Groups}

There were no significant differences between groups on age, gender, and education, except for marital status. As there was no theoretical reason to control for the group differences in marital status, a MANOVA was performed to assess the differences in positive ER strategies between categorized groups. The results showed that Box's M Test of Equality of Covariance is not significant and there are significant differences between groups on the combined dependent variables, $F$ $(6,1060)=29.15, p<.001$, Wilks' $\Lambda=.73$, partial $\eta^{2}=14$. Analysis of covariance result showed that there are significant differences between groups on positive rumination, $F(3$, $531)=18.02, p<.001$, partial $\eta^{2}=.10$, and dampening, $F(3$, $531)=57.57, p<.001$, partial $\eta^{2}=.24$. Follow-up analysis using a Bonferroni adjustment showed that there are significant differences between depression comorbid with GAD and depression, depression comorbid with GAD and GAD, depression comorbid with GAD and the nonclinical group in dampening (depression comorbid with GAD $>$ MDD $>$ GAD $>$ nonclinical) and positive rumination (nonclinical $>\mathrm{GAD}>$ MDD > depression comorbid with GAD). There were also significant differences between the MDD and nonclinical groups in dampening (MDD > nonclinical), but there were no significant differences between GAD and depression, GAD and nonclinical in dampening and positive rumination, and MDD and nonclinical in positive rumination (Table 2).

\section{Discussion}

According to a transdiagnostic approach, the present study sought first, to examine the mediating role of positive ER strategies (dampening and positive rumination) in the relationship between experiential avoidance and deficits in ER with depression and GAD symptoms. Our results showed a significant relationship between all study variables, including deficits in ER, experiential avoidance, dampening, positive rumination, depression, and GAD. Furthermore, the results revealed that dampening and positive rumination mediate the relation between deficits in ER and experiential avoidance with depression and GAD symptoms, which supports our hypothesis. Our results are consistent with previous studies that have demonstrated the role of both positive ER strategies in depression and GAD (Eisner et al., 2009; Nelis et al., 2015), but to our knowledge, this is the first study that examined the mediating role of dampening and positive rumination in depression and GAD symptoms. Our finding supports the notion that ER disturbances, including positive and negative ER disturbances, stem from the same psychological factors that predispose individuals to the development and maintenance of emotional disorders (Carl, Soskin, Kerns, \& Barlow, 2013). Moreover, there is a relationship between positive and negative ER, and there may be a continuum from negative to positive ER, wherein psychological mechanisms respond similarly on this continuum to both positive and negative ER strategies (Johnson \& Wood, 2017). Furthermore, the effectiveness of some psychological interventions, such as mindfulnessbased therapy or the unified protocol for transdiagnostic treatment of emotional disorders, on positive ER corroborates our findings (Carl, Gallagher, \& Barlow, 2018; Geschwind, Martinmäki, \& Garland, 2019).

In our mediation model, deficits in ER were more associated with dampening and positive rumination than experiential avoidance, indicating that this construct has more impact on the psychopathology of depression and GAD through positive ER strategies. One explanation is that deficits in ER and positive ER may have shared underlying process. Another explanation is that individuals may respond to positive emotion just as they respond to negative emotion (Spitzer et al., 2006). In short, individuals who do not accept emotional 
Table 2 Results of MANOVA on Positive Emotion Regulation Strategies

\begin{tabular}{|c|c|c|c|c|c|c|c|c|}
\hline Variables & $\begin{array}{l}\text { Depression } \\
(n=69) M(S D)\end{array}$ & $\begin{array}{l}\text { Depression and GAD } \\
(n=42) M(S D)\end{array}$ & $\operatorname{GAD}(n=28) M(S D)$ & $\begin{array}{l}\text { Nonclinical } \\
(n=396) M(S D)\end{array}$ & $F$ & $d f$ & $\eta^{2}$ & $\begin{array}{l}\text { Significant } \\
\text { Bonferroni }\end{array}$ \\
\hline Positive Rumination & $26.42(5.74)$ & $21.76(5.93)$ & $28.32(5.35)$ & $28.22(5.53)$ & $18.02^{* * *}$ & 3,531 & .10 & $\begin{array}{l}1>2 \\
3>2 \\
4>2\end{array}$ \\
\hline Dampening & $13.11(3.82)$ & $17.95(3.72)$ & $11.89(3.33)$ & $10.44(3.70)$ & $57.57^{* *}$ & 3,531 & .24 & $\begin{array}{l}2>1 \\
2>3 \\
2>4 \\
1>4\end{array}$ \\
\hline
\end{tabular}

Note. 1 = Depression; 2 = Depression and GAD; $3=\mathrm{GAD} ; 4=$ Nonclinical

$* * p<.001$

responses have difficulties engaging in goal directed behaviors, difficulties with impulse control, lack of emotional awareness, limited access to ER strategies, and lack of emotional clarity, and they might tend to use more dampening and less positive rumination in response to positive emotion.

The second aim of the current study was to evaluate the distinct and shared roles of dampening and positive rumination in GAD, depression, and depression comorbid with GAD clinical groups and a nonclinical group. The results indicate that participants differed in the use of these strategies. With regard to dampening, the comorbid group obtained the highest scores and the nonclinical the lowest, with the depression and generalized anxiety groups being the middle-point, without differences among them. A reverse pattern of differences was observed in the case of positive rumination; nonclinical participants had the highest scores and the comorbid group the lowest. Both depression and GAD groups scored higher than the comorbid group. Results are consistent with previous studies indicating that dampening is more closely associatedwith anxiety and depression symptoms than positive rumination (Abasi et al., 2018; Olofsson, Boersma, Engh, \& Wurm, 2014). Taken together, the path analysis findings showing the relation between dampening and positive rumination with depression and GAD and the MANOVA results indicate that dampening and somehow positive rumination may be transdiagnostic constructs that occur with varying severity across emotional disorders (Carl et al., 2013; Eisner et al., 2009; Gruber, 2019), with dampening being higher in more severe disorders such as depression comorbid with GAD and positive rumination being higher in less severe pathological groups such as GAD and nonclinical participants. These results indicate that dampening can be considered a maladaptive positive ER strategy, whereas positive rumination may be considered an adaptive positive ER strategy. On the other hand, our finding on positive rumination contradicts a recent investigation (Cludius et al., 2020), which concluded that positive rumination might not be a shared regulatory process across a range of mental disorders.

Our findings suggest several implications that benefit assessment, prevention, and treatment of emotional disorders. First, they highlight the need to consider both negative and positive ER in understanding the nature and underlying mechanisms of depression and GAD. Second, they indicate a mechanism in which positive ER strategies may contribute to emotional disorders. Finally, the transdiagnostic entities of dampening and somehow positive rumination suggest the likely utility of a transdiagnostic conceptualization and specific positive ER strategies in the prevention and treatment of emotional disorders.

This study has several strengths, including examining the mediating role of positive ER in the psychopathology of GAD and depression, and examining the different roles of positive ER strategies in depression comorbid with GAD. Nonetheless, some limitations should be considered. A noteworthy limitation is reliance on self-report assessment in evaluating trait positive ER; experience-sampling methodologies that assess state and multiple levels of analysis are recommended. Although the use of nonclinical samples is common in exploratory studies dealing with a scarcely investigated topic, it is necessary to replicate the results in clinical populations. The use of cut-off points to categorize individuals into clinical groups is another limitation. Moreover, our study was crosssectional; thus, causal conclusions regarding the differences between groups in dampening and positive rumination could not be made. In addition, although our findings support the adaptive use of positive rumination and maladaptive use of dampening, the context for the use of positive ER is not clear and it is possible that in different contexts, dampening and positive rumination may have varied impact on psychopathology and mental health (Bonanno \& Burton, 2013). Finally, more research is needed to uncover both the specific and transdiagnostic role of positive ER. 


\section{Conclusion}

The current study provides preliminary evidence for the mediating role of positive ER strategies in the relationship between deficits in ER and experiential avoidance with depression and GAD symptoms. Additionally, dampening was more associated with the clinical groups than positive rumination, suggesting that this strategy could be more clearly considered as a transdiagnostic factor. Our findings support the transdiagnostic role of positive ER strategies in predisposing individuals to emotional disorders, suggesting that there may be a psychological pathway similar to negative ER in the relationship between underlying cognitive and emotional mechanisms (deficits in ER and experiential avoidance) with emotional disorders. Future studies with clinical samples must support this suggestion.

Funding This work was supported by the Tehran University of Medical Sciences [grant number: 96-02-30-34239.

Data Availability Data are available upon request from the authors.

\section{Compliance with Ethical Standards}

Conflicts of Interest/Competing Interests On behalf of all authors, the corresponding author states that there is no conflict of interest.

Code Availability Not applicable.

\section{References}

Abasi, E., Fti, L., Molodi, R., \& Zarabi, H. (2013). Psychometric properties of Persian version of acceptance and action questionnaire-II. Journal of Psychological Models and Methods, 3(10), 65-80.

Abasi, I., Feldman, G., Farazmand, S., Pourshahbaz, A., \& Sarichloo, M. E. (2018). A psychometric evaluation of Iranian version of the responses to positive affect (RPA) questionnaire. Iranian Journal of Psychiatry and Behavioral Sciences, 12(4), e11923. https://doi.org/ 10.5812/ijpbs.11923.

Arbuckle, J. L. (2014). Amos 23.0 User's Guide. Chicago: IBM SPSS.

Asgari, P., Pasha, G. R., \& Aminiyan, M. (2009). Relationship between emotion regulation, mental stresses and body image with eating disorders of women. Applied Psychology, 4(5), 65-78.

Beck, A. T., Steer, R. A., \& Carbin, M. G. (1988). Psychometric properties of the Beck depression inventory: Twenty-five years of evaluation. Clinical Psychology Review, 8(1), 77-100. https://doi.org/10. 1016/0272-7358(88)90050-5.

Bonanno, G. A., \& Burton, C. L. (2013). Regulatory flexibility: An individual differences perspective on coping and emotion regulation. Perspectives on Psychological Science, 8(6), 591-612. https://doi. org/10.1177/1745691613504116.

Bonanno, G. A., Pat-Horenczyk, R., \& Noll, J. (2011). Coping flexibility and trauma: The perceived ability to cope with trauma (PACT) scale. Psychological Trauma: Theory, Research, Practice, and Policy, 3(2), 117-129. https://doi.org/10.1037/a0020921.

Bond, F. W., Hayes, S. C., Baer, R. A., Carpenter, K. M., Guenole, N., Orcutt, H. K., Waltz, T., \& Zettle, R. D. (2011). Preliminary psychometric properties of the acceptance and action questionnaire-II:
A revised measure of psychological inflexibility and experiential avoidance. Behavior Therapy, 42(4), 676-688. https://doi.org/10. 1016/j.beth.2011.03.007.

Carl, J. R., Soskin, D. P., Kerns, C., \& Barlow, D. H. (2013). Positive emotion regulation in emotional disorders: A theoretical review. Clinical Psychology Review, 33(3), 343-360. https://doi.org/10. 1016/j.cpr.2013.01.003.

Carl, J. R., Gallagher, M. W., \& Barlow, D. H. (2018). Development and preliminary evaluation of a positive emotion regulation augmentation module for anxiety and depression. Behavior Therapy, 49(6), 939-950. https://doi.org/10.1016/j.beth.2017.11.008.

Chapman, A. L. (2019). Borderline personality disorder and emotion dysregulation. Development and Psychopathology, 31(3), 11431156. https://doi.org/10.1017/S0954579419000658.

Chawla, N., \& Ostafin, B. (2007). Experiential avoidance as a functional dimensional approach to psychopathology: An empirical review. Journal of Clinical Psychology, 63(9), 871-890. https://doi.org/10. $1002 /$ jclp.20400.

Cludius, B., Mennin, D., \& Ehring, T. (2020). Emotion regulation as a transdiagnostic process. Emotion, 20(1), 37-42. https://doi.org/10. 1037/emo0000646.

Dixon, M. L., Moodie, C. A., Goldin, P. R., Farb, N., Heimberg, R. G., \& Gross, J. J. (2020). Emotion regulation in social anxiety disorder: Reappraisal and acceptance of negative self-beliefs. Biological Psychiatry: Cognitive Neuroscience and Neuroimaging, 5(1), 119-129. https://doi.org/10.1016/j.bpsc.2019.07.009.

Dodd, A., Lockwood, E., Mansell, W., \& Palmier-Claus, J. (2019). Emotion regulation strategies in bipolar disorder: A systematic and critical review. Journal of Affective Disorders, 246, 262-284. https://doi.org/10.1016/j.jad.2018.12.026.

Dryman, M. T., \& Heimberg, R. G. (2018). Emotion regulation in social anxiety and depression: A systematic review of expressive suppression and cognitive reappraisal. Clinical Psychology Review, 65, 1742. https://doi.org/10.1016/j.cpr.2018.07.004.

du Pont, A., Welker, K., Gilbert, K. E., \& Gruber, J. (2016). The emerging field of positive emotion dysregulation. In K. D. Vohs \& R. F. Baumeister (Eds.), Handbook of self-regulation: Research, theory and applications (3rd ed., pp. 364-379). New York, NY: Guilford Press.

Eisner, L. R., Johnson, S. L., \& Carver, C. S. (2009). Positive affect regulation in anxiety disorders. Journal of Anxiety Disorders, 23(5), 645-649. https://doi.org/10.1016/j.janxdis.2009.02.001.

Feldman, G. C., Joormann, J., \& Johnson, S. L. (2008). Responses to positive affect: A self-report measure of rumination and dampening. Cognitive Therapy and Research, 32(4), 507-525. https://doi.org/ 10.1007/s10608-006-9083-0.

Fredrickson, B. L., \& Branigan, C. (2005). Positive emotions broaden the scope of attention and thought-action repertoires. Cognition \& Emotion, 19(3), 313-332. https://doi.org/10.1080/ 02699930441000238.

George, D., \& Mallery, P. (2016). IBM SPSS statistics 23 step by step: A simple guide and reference. New York, NY: Routledge. https://doi. org/10.4324/9781315545899.

Geschwind, N., Martinmäki, S., \& Garland, E. L. (2019). Facilitation of positive emotions through mindfulness-based therapy. In J. Gruber (Ed.), The Oxford handbook of positive emotion and psychopathology (pp. 1110-1124). New York, NY: Oxford University Press.

Ghassemzadeh, H., Mojtabai, R., Karamghadiri, N., \& Ebrahimkhani, N. (2005). Psychometric properties of a Persian-language version of the Beck depression inventory-second edition: BDI-II-PERSIAN. Depression and Anxiety, 21(4), 185-192. https://doi.org/10.1002/ da. 20070

Gratz, K. L., \& Roemer, L. (2004). Multidimensional assessment of emotion regulation and dysregulation: Development, factor structure, and initial validation of the difficulties in emotion regulation scale. 
Journal of Psychopathology and Behavioral Assessment, 26(1), 4154. https://doi.org/10.1023/B:JOBA.0000007455.08539.94.

Gruber, J. (2019). The Oxford handbook of positive emotion and psychopathology. New York, NY: Oxford University Press. https://doi.org/10.1093/oxfordhb/9780190653200.001.0001.

Hayes, S. C. (2004). Acceptance and commitment therapy, relational frame theory, and the third wave of behavioral and cognitive therapies. Behavior Therapy, 35(4), 639-665. https://doi.org/10.1016/ S0005-7894(04)80013-3.

Hayes, S. C., Strosahl, K., Wilson, K. G., Bissett, R. T., Pistorello, J., Toarmino, D., Polusny, M. A., Dykstra, T. A., Batten, S. V., Bergan, J., Stewart, S. H., Zvolensky, M. J., Eifert, G. H., Bond, F. W., Forsyth, J. P., Karekla, M., \& McCurry, S. M. (2004). Measuring experiential avoidance: A preliminary test of a working model. The Psychological Record, 54(4), 553-578. https://doi.org/10.1007/ $\mathrm{BF} 03395492$.

Johnson, J., \& Wood, A. M. (2017). Integrating positive and clinical psychology: Viewing human functioning as continua from positive to negative can benefit clinical assessment, interventions and understandings of resilience. Cognitive Therapy and Research, 41(3), 335-349. https://doi.org/10.1007/s10608-015-9728-y.

Kirk, A., Meyer, J. M., Whisman, M. A., Deacon, B. J., \& Arch, J. J. (2019). Safety behaviors, experiential avoidance, and anxiety: A path analysis approach. Journal of Anxiety Disorders, 64, 9-15. https://doi.org/10.1016/j.janxdis.2019.03.002.

Meyers, L. S., Gamst, G., \& Guarino, A. J. (2016). Applied multivariate research: Design and interpretation ( $3 r d$ ed.). Thousand Oaks, CA: Sage Publications.

Moskowitz, J. T., Epel, E. S., \& Acree, M. (2008). Positive affect uniquely predicts lower risk of mortality in people with diabetes. Health Psychology, 27(1S), S73-S82. https://doi.org/10.1037/0278-6133. 27.1.S73.

Nelis, S., Holmes, E. A., \& Raes, F. (2015). Response styles to positive affect and depression: Concurrent and prospective associations in a community sample. Cognitive Therapy and Research, 39(4), 480491. https://doi.org/10.1007/s10608-015-9671-y.

O’Toole, M. S., Renna, M. E., Mennin, D. S., \& Fresco, D. M. (2019). Changes in decentering and reappraisal temporally precede symptom reduction during emotion regulation therapy for generalized anxiety disorder with and without co-occurring depression. Behavior Therapy, 50(6), 1042-1052. https://doi.org/10.1016/j. beth.2018.12.005.

Olofsson, M. E., Boersma, K., Engh, J., \& Wurm, M. (2014). A psychometric evaluation of the Swedish version of the responses to positive affect questionnaire. Nordic Journal of Psychiatry, 68(8), 588-593. https://doi.org/10.3109/08039488.2014.898792.
Omani-Samani, R., Maroufizadeh, S., Ghaheri, A., \& Navid, B. (2018). Generalized anxiety Disorder-7 (GAD-7) in people with infertility: A reliability and validity study. Middle East Fertility Society Journal, 23(4), 446-449. https://doi.org/10.1016/j.mefs.2018.01. 013.

Rand, D. G., Kraft-Todd, G., \& Gruber, J. (2015). The collective benefits of feeling good and letting go: Positive emotion and (dis) inhibition interact to predict cooperative behavior. PLoS One, 10(1), 1-12. https://doi.org/10.1371/journal.pone.0117426.

Seager, I., Mennin, D. S., \& Aldao, A. (2019). Positive emotion in generalized anxiety disorder. In J. Gruber (Ed.), The Oxford handbook of positive emotion and psychopathology (pp. 298-311). New York, NY: Oxford University Press. https://doi.org/10.1093/oxfordhb/ 9780190653200.013.20

Silton, R. L., Kahrilas, I. J., Skymba, H. V., Smith, J., Bryant, F. B., \& Heller, W. (2020). Regulating positive emotions: Implications for promoting well-being in individuals with depression. Emotion, 20(1), 93-97. https://doi.org/10.1037/emo0000675.

Sloan, E., Hall, K., Moulding, R., Bryce, S., Mildred, H., \& Staiger, P. K. (2017). Emotion regulation as a transdiagnostic treatment construct across anxiety, depression, substance, eating and borderline personality disorders: A systematic review. Clinical Psychology Review, 57, 141-163. https://doi.org/10.1016/j.cpr.2017.09.002.

Spinhoven, P., Drost, J., de Rooij, M., van Hemert, A. M., \& Penninx, B. W. (2014). A longitudinal study of experiential avoidance in emotional disorders. Behavior Therapy, 45(6), 840-850. https://doi.org/ 10.1016/j.beth.2014.07.001.

Spitzer, R. L., Kroenke, K., Williams, J. B., \& Löwe, B. (2006). A brief measure for assessing generalized anxiety disorder: The GAD-7. Archives of Internal Medicine, 166(10), 1092-1097. https://doi. org/10.1001/archinte.166.10.1092.

Williams, M. J., Dalgleish, T., Karl, A., \& Kuyken, W. (2014). Examining the factor structures of the five facet mindfulness questionnaire and the self-compassion scale. Psychological Assessment, 26(2), 407-418. https://doi.org/10.1037/a0035566.

Wood, J. V., Heimpel, S. A., \& Michela, J. L. (2003). Savoring versus dampening: Self-esteem differences in regulating positive affect. Journal of Personality and Social Psychology, 85(3), 566-580. https://doi.org/10.1037/0022-3514.85.3.566.

Zhou, Y., Cao, Z., Yang, M., Xi, X., Guo, Y., Fang, M., et al. (2017). Comorbid generalized anxiety disorder and its association with quality of life in patients with major depressive disorder. Scientific Reports, 7(1), 1-8. https://doi.org/10.1038/srep40511.

Publisher's Note Springer Nature remains neutral with regard to jurisdictional claims in published maps and institutional affiliations. 